\title{
The Fourth Asian Radiology Summit
}

\author{
Current Projects of the Radiological Societies in Asia
}

Noriyuki Tomiyama $^{1} \cdot$ Kei Yamada ${ }^{2} \cdot$ Yoshiyuki Watanabe $^{3} \cdot$ Yutaka Imai $^{4}$

Published online: 16 March 2020

( ) Japan Radiological Society 2020

\section{Introduction}

The Japan Radiological Society (JRS) successfully held the fourth Asian Radiological on April 12, 2019 in Yokohama, Japan. It was attended by the Presidents of participating Asian radiological societies. The delegates presented and discussed the current projects of their respective societies with emphasis on their priorities and status of their on-going projects. It was a great opportunity for the societies to have a closer look on pressing issues and needs that are being addressed and how they are being addressed locally and globally.

The participating societies included the Taiwan Radiological Society (TRS), the Korean Society of Radiology (KSR), the Philippine College of Radiology (PCR), the Mongolian Society of Radiology (MSR), the Singapore Radiological Society (SRS), the Indian Radiological Imaging Association (IRIA), the Radiological Society of Thailand (RST), the Radiological Society of Pakistan (RSP), and the Sri Lanka College of Radiologists (SLCR), and the Japan Radiological Society (JRS). Figure 1 is a photo of the delegates to the 4 th Asian Radiology Summit.

Noriyuki Tomiyama

tomiyama@radiol.med.osaka-u.ac.jp

1 Department of Radiology Graduate School of Medicine, Osaka University, Osaka, Japan

2 Department of Radiology, Kyoto Prefectural University of Medicine, Kyoto, Japan

3 Department of Radiology, Shiga University of Medical Science, Otsu, Japan

4 Department of Radiology, Tokai University School of Medicine, Isehara, Japan

\section{Taiwan Radiological Society}

The increased number of breast and lung cancer in Taiwan urged the TRS to focus on the improvement of mammography image quality, staff training, and the launching of the Taiwan Lung Cancer Screening for Never Smoker Trial (TALENT) project.

The TRS's main goal is to detect breast cancer at an earlier stage. Women in Taiwan receive a free mammogram every two years. The number of screening and hospitals increased and screening cases continue to rise every year. About $40 \%$ of radiologists are actively participating in this project.

Another priority project of Taiwan is the Taiwan Lung Cancer Screening for Never Smoker Trial (TALENT). This was prompted by the downward trend of cigarette smoking rate among adults and upward trend of lung cancer incidence.

There is a never-smoker predominance of $55 \%$ and adenocarcinoma predominance of $70 \%$. Lung adenocarcinoma epidermal growth factor receptor (EGFR) mutation is $60 \%$ and stages III/IV are more than $60 \%$ in both smokers and never-smokers.

It is Taiwan's aim to research strategies of screening for early detection and hopefully, prevention.

\section{Korean Society of Radiology}

The KSR aims to look at both medicine and IT with balance, follow scientific principles and evidence, provide correct information to the public, educate radiologists as well as other stakeholders, make reasonable policy suggestions, and build a collaborative partnership between the industry and the government. In doing so, KSR has started working with policy makers regarding AI-related government policy. 
Fig. 1 ARS 2019 delegates. From the left on the first row: Dr. Jitjaroen Chaiyakum, Dr. Eva De Leon, Dr. Muhammad Hamid Akram, Prof. Yutaka Imai, Dr. Hemant Patel, Dr. Joo Hyeong Oh, and Dr. Dagvasumberel Gonchigsuren. From the left, second row: Dr. Andrew Tan, Dr. Prasad De Silva, Prof. Kei Yamada, Prof. Noriyuki Tomiyama, and Dr. YeunChung Chang

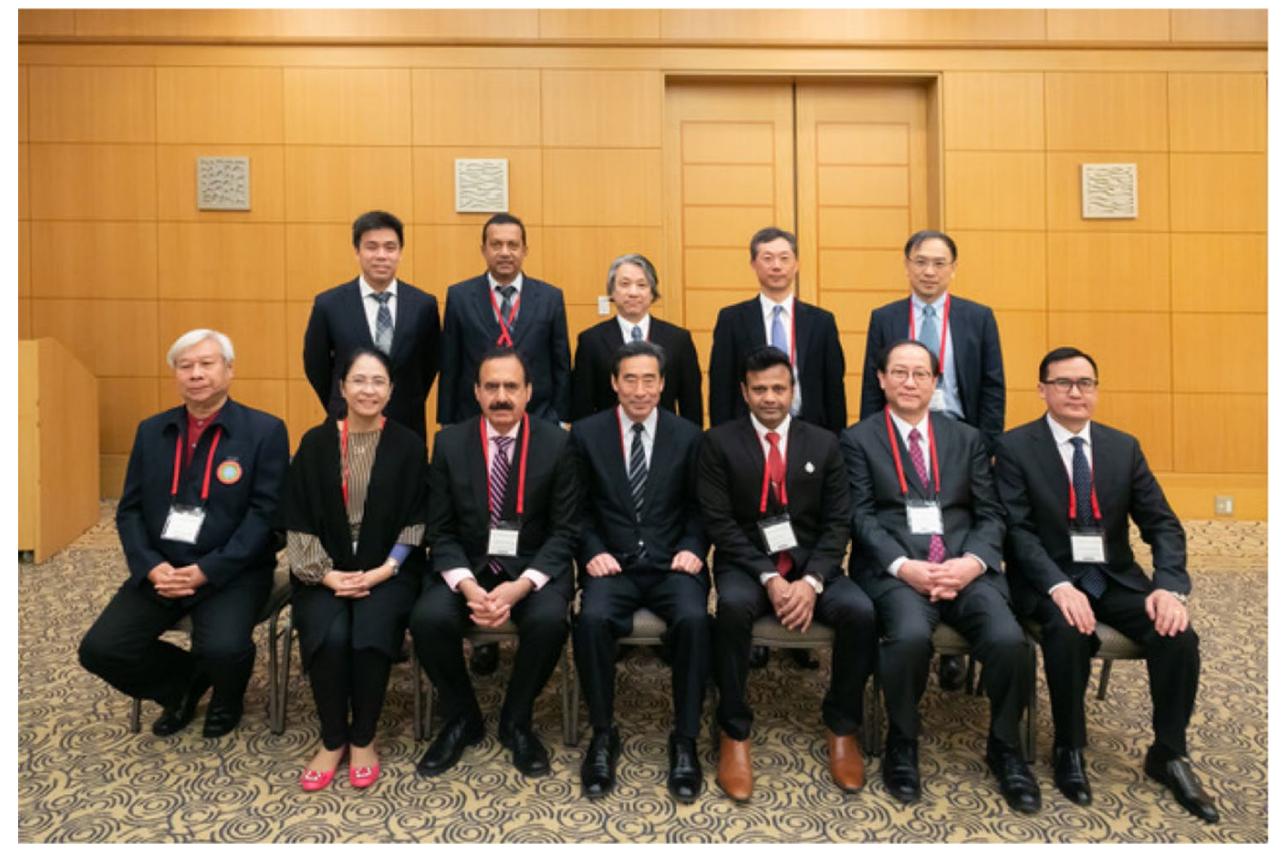

Guidelines regarding the evaluation and requirements for health insurance coverage of radiology AI software device are now being made. Consultations and collaboration with the national Health Insurance Review and Assessment Service (HIRA) and National Evidence-based Healthcare Collaborating Agency (NECA) are on-going.

The KSR is also focused on the reformation of their competency-based training program. The Korean Association of Medical Science and 26 Korean Societies of specialties will reform their residency training program and establish evaluation tools to train good qualified specialists. To do so, appropriate evaluation of training facilities and supports of hospitals and the level of achievement of individual trainee is the key element, hence, the reformations on training program renewal and evaluation training.

The KSR is now also reinforcing their capacity to respond to national healthcare policies. Korea has only a single medical insurance system run by the government- the National Health Insurance Service (NHIS). The NHIS only covers about two thirds of medical costs. For insured services, $25 \%$ of the fee should be paid by the patients. About $10 \%$ of medical services are uninsured, including MRI and ultrasonography (USG). Therefore, costs paid by the patients themselves are relatively high.

These caused some challenges for the KSR. One of which is cutting the fee for radiology services. Rapid growth of medical imaging has put pressure on the budget of NHIS. As a result, fees for radiological services have been reduced by $20-40 \%$ in the last decade.

Another is the Moon care proposed by Mr. President Moon Jae-Ihn. It is a policy for widening the coverage range of NHIS to try to cover all the MRI and USG examinations by NHIS, which were uninsured in the past. Fees for insured services are much cheaper than that of uninsured services; therefore, fees for MRI and USG examinations have been cut down.

As a response to these challenges, the KSR is putting in the effort to maintain or even raise the fee for radiology services. The workload of radiologists in radiology services is underestimated and efforts for quality assurance and management of radiological department are not rewarded. The KSR is trying to raise doctor's fee for radiology service ("fee for imaging interpretation") and establish the fee for quality assurance.

Separating doctor's fee from hospital fee for radiological services can also be a solution. According to the NHIS, doctor's fee and hospital fee are not separated. Radiology services are considered as "medical tests" and not "doctor's act." By separating doctor's fee that is, fee for imaging interpretation, from the fee for imaging acquisition, efforts of doctor-radiologists can be properly rewarded.

\section{Philippine College of Radiology}

The PCR is now focused on further expansion of the scope and extent of the society by creation of more chapters in the country. The Philippines is made up of many small islands, so expansion and creation of more chapters will ease communication and information dissemination.

They are also focused in further creation of specialty interest groups to concentrate on system-based education and training such as the Philippine Radiologic Society of Nuclear Medicine and Molecular Imaging, Diagnostic 
Breast Imaging Society, Neuroradiology, Pediatric Radiology, and Skeletal Radiology Society of the Philippines.

The residency and fellowship-based training in the Philippines is also currently in transition from a modality-based approach to a system-based approach.

\section{Mongolian Society of Radiology}

The E-Health Project is a priority program in Mongolia. The objective is to improve integration and utilization of health information and E-Health solutions for better health service delivery. The key components of this project are clinical data collection, access, and sharing. Mongolia aims to establish a solid basis for standardized data and medical image exchange between healthcare providers and users, develop the mechanism for such data exchange to take place, and pilot test this mechanism in selected sites and that's where the picture archiving and communication system (PACS) project comes in.

The first step of this project is the integration of five pilot hospitals' digital imaging workflow to the central PACS. This includes upgrading of selected pilot facilities to be able to use and store digitally acquired images (mini PACS) and upgrading of local networks to be able to share digital images with the central PACS.

Images are digitized at the point of acquisition, sent and archived in local mini PACS and in the central PACS, and viewed from the PACS by the healthcare professionals. Hospitals and patients, health care institutions, and health policy and decision makers will directly benefit from this project. A health database is now established for all citizens, duplicate radiology, and laboratory examinations will be prevented. Doctor's appointments and test results are now conducted electronically.

Another priority project focuses on the improvement of diagnostic ability in the early phase of pneumoconiosis and asbestos-related respiratory disease. The purpose is to be able to make an early diagnosis of pneumoconiosis and asbestos-exposure-related respiratory disease.

The timeframe is from March 2018 to March 2021 and the expected outcomes include increased number of radiologists and pathologists who have a specific diagnostic ability about the pneumoconiosis and asbestos-related respiratory disease, increased number of radiology technologists who can make high-quality radiographic image and increased number of pathology technologists who make pathological specimens with high quality including immuno-histochemical staining.

The MSR is also working to establish a network of radiologists in Mongolia and a consultation system of pathological and radiological diagnosis between Mongolia and Japan using the telemedicine.

\section{Singapore Radiological Society}

In Singapore, a decision was made in 2015 to develop and grow the Singapore Radiological Society Annual Scientific Meeting (SRS ASM) into a prominent regional scientific meeting. To give Singapore and regional radiologists' access to a world-class imaging conference, they started partnership with a professional events company in 2015 to develop a conference now known as the RadiologyAsia.

2019 Singapore Congress of Radiology focuses on workshops in Interventional Radiological Education Singapore (WIRES). For the coming year, the 19th Congress of the ASEAN Association of Radiology Goals are to develop a world-class scientific conference and to attract a regional delegate count of 2000 participants by 2020 .

Singapore Radiological Society ASEAN Scholarship and Conference Prizes is another initiative by the SRS. Started in 2016, it aims to provide cash rewards to top scientific posters and presentations done by junior radiologists, to provide conference sponsorship for a number of ASEAN junior radiologists to attend the SRS ASM, to expand and include local hospitality in the future, and to provide opportunities to deserving junior radiologists in the region. The SRS aims to remain active in industry and social engagements through these projects to develop and grow the SRS.

\section{Indian Radiological Imaging Association}

The IRIA's priority project is geared towards social and academic development. The IRIA aims to be more socially and academically active through outreach programs. They are active in organizing continuing medical education (CMEs) at various state and district levels with the help of local subchapters so that radiology teaching reaches every corner of the country. This year's primary focus is interventional radiology and breast imaging.

The second project of the IRIA is the Youth Wing of Radiology (YUVA) IRIA. It was launched to exclusively cater to the welfare of postgraduate residents and young radiologists. It was formed to not only understand and address the problems faced by radiology residents and young radiologists, but also to showcase the talent of younger radiologists.

The third is Raksha IRIA. It is a project that aims to empower girls and women, to give them importance in the society. It aims to provide a platform where the members can help in the current social issues centered on the "girl child" and to bring together the members on a social platform. 


\section{Radiological Society of Thailand}

Thailand is also active in their outreach programs giving refresher courses and visiting their provinces.

They host the national annual scientific radiology meeting with the Royal College of Radiologists of Thailand (RCRT). This is to continuously encourage active participation of all related associations, societies, and clubs.

Thailand's National Practice Guidelines are in the works now. The RST has started creating the guidelines, particularly, guidelines for emergency CT brain and radiographs in extremity trauma.

\section{Radiological Society of Pakistan}

In Pakistan, research on finding epidemiological and molecular determinants of breast cancer is a priority of the RSP. Their target subjects are mainly the population of rural and urban areas of the Federal Capital Territory. Their methodology includes mammography screening, biopsies for definitive diagnosis, genetic analysis of proven cases, and gene mutation analysis.

The second priority project is the development of protocols for breast cancer care. A large group of specialists from all disciplines related to breast cancer care in Pakistan are working to develop protocols specific to Pakistan. The specialized group includes, diagnostic radiologists, surgeons, oncologists, pathologists, physicists, and rehabilitation specialists.

The RSP also built a center for diagnosis and treatment of vascular anomalies in children where radiological diagnosis and assessment, sclerotherapy, and surgical management have been carried on 160 patients in the last 9 months.

\section{Sri Lanka College of Radiologists}

Just like Mongolia, Sri Lanka is also working on a unified database. Sri Lanka's PACS project aims to connect 24 centres island wide. Their main server is situated at the National Hospital of Sri Lanka (NHSL).

Sri Lanka also started a project on mechanical clot retrieval for acute stroke in February 2018. To date, they have had 16 successful cases and the count continues to rise.

In 2017, microwave ablation was started. It has been performed on many cases but mostly on hepatocellular carcinoma and liver metastases and continues to show great success.

\section{Japan Radiological Society}

JapanSafe is a priority project of the JRS. The objective is to implement a structural reform to optimize the distribution of medical resources and to improve safety, efficiency, standardization, and optimization of medical imaging in Japan. One of the key components of this project is clinical decision support.

In the United States, the Protecting Access to Medicare Act (PAMA) requires referring providers to consult appropriate use criteria (AUC) prior to ordering advanced diagnostic imaging services (ADIS)—CT, MR, nuclear medicine, and PET_-for Medicare patients, starting Jan 1, 2020.

To follow suit, the JRS aims to install information systems in hospitals. The interactive communication system provides evidence-based guidelines to assist referring physicians in making the most appropriate use of medical imaging.

The JRS is also focused on addressing some challenges related to the development and application of AI to radiology in clinical medicine. A popular sentiment that radiologists could lose their roles to AI has caused the decreasing number of applicants in radiology. To address this issue, the JRS actively supports the positive promotion of AI. It highlights that AI can secure objectivity with its quantitative analysis, reduce human error, be used for medical education and instead of threaten, and AI can actually enhance radiologist's career.

Lastly, in relation to the speedy development of AI, the JRS is obliged to educate the public on the importance of radiologists' job in modern clinical medicine with emphasis on the radiologists' ability not only in diagnosing but also in selecting the most appropriate treatment for individual patients. $\mathrm{AI}$ is a useful tool for radiologists and radiologists remain indispensable in verifying the accuracy and errors of AI.

Acknowledgements This paper was written based on the presentations at the Asian Radiology Summit 2019 held during the JRC 2019 by the following representatives of the participating societies (in order of presentation): Yeun-Chung Chang, MD (Taiwan Radiological Society), Joo Hyeong Oh, MD (Korean Society of Radiology), Eva De Leon, MD (Philippine College of Radiology), Dagvasumberel Gonchigsuren, MD (Mongolian Radiological Society), Andrew Tan, MD (Singapore Radiological Society), Hemant Patel, MD (Indian Radiological and Imaging Association), Khalilur Rahman, MD (Bangladesh Society of Radiology and Imaging), Lilian Leong, MD (Hong Kong College of Radiologists), Khin Hla, MD (Myanmar Radiological Society), Ansari Mukhtar Alam, MD (Nepal Radiologist Association), Jitjaroen Chaiyakum, MD (Radiological Society of Thailand), Muhammad Hamid Akram, MD (Radiological Society of Pakistan), Prasad De Silva, MD (Sri Lanka College of Radiologists), and Yutaka Imai, MD, PhD (Japan Radiological Society). We thank Miss Liza Mia Christine F. Maquiraya for editing this manuscript.

Publisher's Note Springer Nature remains neutral with regard to jurisdictional claims in published maps and institutional affiliations. 